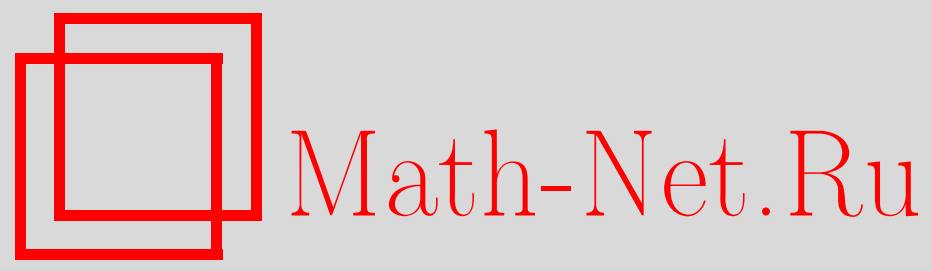

А. Л. Шабунин, Примеры $\alpha$-полных систем $k$-значной логики при $k=3,4$, Дискрет. матем., 2006, том 18, выпуск $4,45-55$

DOI: https://doi.org/10.4213/dm72

Использование Общероссийского математического портала Math-Net.Ru подразумевает, что вы прочитали и согласны с пользовательским соглашением http://www mathnet.ru/rus/agreement

Параметры загрузки:

IP : 3.85 .7 .115

26 апреля 2023 г., 18:32:28

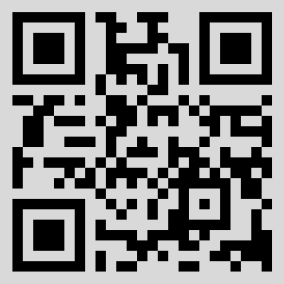




\title{
Примеры $\alpha$-полных систем $k$-значной логики при $k=3,4$
}

\author{
() 2006 г. А. Л. Шабунин
}

\begin{abstract}
В статье доказывается $\alpha$-полнота конечных систем функций $k$-значной логики при $k=3,4$, содержащих все подстановки из симметрической группы $S_{k}$ подстановок множества $E_{k}=\{0,1, \ldots, k-1\}$, операцию сложения по модулю $k$ и еще $k$ фиксированных бинарных операций. Этот результат затем распространяется на некоторые другие системы функций, получающиеся из указанных заменой операции сложения на квазигрупповую операцию.
\end{abstract}

Пусть $P_{\boldsymbol{k}}$ - множество всех функщий $k$-значной логики, $X$ - множество символов переменных со значениями из $E_{k}=\{0,1, \ldots, k-1\}, P_{k}(n)-$ множество всех функций из $P_{k}$, зависящих от переменных $x_{1}, x_{2}, \ldots, x_{n}$.

Следуя М. М. Глухову [1], определим индуктивно понятие $\alpha$-формулы над непустым классом $F \subseteq P_{k}$ как выражения вида $f\left(\varphi, x_{i_{1}}, \ldots, x_{i_{s}}\right)$, где $f\left(x_{1}, \ldots, x_{s+1}\right)$ - обозначение функции из $F, \varphi$ - символ переменной или $\alpha$-формула над $F$, а $x_{i_{1}}, \ldots, x_{i_{s}} \in X$. Множество всех функций из $P_{k}$, реализуемых (представимых) $\alpha$-формулами над $F$, называется $\alpha$-пополнением ( $\alpha$-замыканием) множества $F$ и обозначается через $[F]_{\alpha}$. При построении $\alpha$-формул используется ограниченная суперпозиция: $\alpha$-формула может подставляться в $f\left(x_{1}, \ldots, x_{s+1}\right)$ только вместо $x_{1}$. Такие суперпозиции называются $\alpha$-суперпозициями. Если $[F]_{\alpha}=P_{k}$, то $F$ называется $\alpha$-полной системой; $\alpha$-полная система функций $F$ называется $\alpha$-базисом $P_{k}$, если $F \backslash\{f\}$ уже не является $\alpha$-полной при любой $f \in F$. В [1] доказано, что $\alpha$-полной при $k \geqslant 7$ является любая система функций из $P_{k}$, содержащая все подстановки из симметрической группы $S_{k}$ подстановок множества $E_{k}$ и одну любую квазигрупповую функщию. В [2] приведены условия $\alpha$-полноты систем функций $k$-значной логики, состоящих из функций, у которых все одноместные подфункщии, полученные произвольной фиксацией всех переменных, кроме первой, являются подстановками; для $k \geqslant 5$ построены $\alpha$-полные системы из двух бинарных операций с правым сокращением, а для $k=2$ доказано отсутствие конечных $\alpha$-полных систем. Там же доказано, что при любом $k$ в $P_{k}$ отсутствуют $\alpha$-полные системы, состоящие из одной функции.

В настоящей статье рассматриваются случаи $k=3,4$. Доказывается, что при этих значениях $k$ система функщий $k$-значной логики, содержащая все подстановки множества $E_{k}$, операцию сложения по модулю $k$ и $k$ бинарных операций, определенных ниже, $\alpha$-полна. Этот результат затем распространяется на другие системы функций, содержащие все подстановки множества $E_{k}$, одну квазигрупповую операцию и упомянутые $k$ бинарные операции.

Далее, если не оговорено противное, символы,+- и - обозначают операции сложения, вычитания и умножения по модулю $k$, определенные на множестве $E_{k}$. 
Рассмотрим сначала случай $k=4$.

Лемма 1. Справедливо равенство

$$
\left[x_{1}+x_{2}\right]_{\alpha}=\left[x_{1}+x_{2}, x_{1}-x_{2}\right]_{\alpha} .
$$

Доказательство. Достаточно заметить, что функция $x_{1}-x_{2}$ представляется $\alpha$-формулой $\left(\left(x_{1}+x_{2}\right)+x_{2}\right)+x_{2}$ над системой $\left\{x_{1}+x_{2}\right\}$.

Определим на множестве $E_{4}$ бинарные операции о $o_{i} i=0,1,2,3$, полагая $1 \circ_{0} 2=3$, $3 \circ_{0} 2=1,1 \circ_{1} 3=3,3 \circ_{1} 3=1,1$ о $_{2} 0=3,3$ о $_{2} 0=1,1$ о $_{3} 1=3,3$ о $_{3} 1=1$ и $a \circ_{i} b=a$ в остальных случаях.

Операции о $i$ являются операциями с правым сокращением, то есть удовлетворяют условию

$$
a \circ_{i} c=b \circ_{i} c \Longrightarrow a=b .
$$

Поэтому для них определены правые обратные операции $/ i$, где

$$
a / i b=c \Longleftrightarrow a=c \circ_{i} b \text {. }
$$

Легко проверить, что в нашем случае $x / i y=x \circ_{i} y$.

Пусть

$$
T=S_{4} \cup\left\{+, \circ_{0}, \circ_{1}, \circ_{2}, \circ_{3}\right\} .
$$

Условимся в виде $f h$ записывать суперпозицию $h(f)$ для $f \in P_{4}$ и $h \in S_{4}$. Операцию + будем иногда обозначать символом $\circ_{4}$, а (правую) обратную к ней операцию вычитания - символом /4. Справедливы равенства

$$
x \circ_{4} y=x+y, \quad x / 4 y=x-y=((x+y)+y)+y .
$$

Поэтому любая $\alpha$-формула над $T$ будет иметь вид

$$
\left(\ldots\left(x_{i_{1}} h_{1} \circ_{j_{2}} x_{i_{2}}\right) h_{2} \ldots \circ_{j_{l}} x_{i_{l}}\right) h_{l} \text {, }
$$

где $x_{i_{1}}, \ldots, x_{i_{l}} \in X, h_{1}, \ldots, h_{l} \in S_{4}, j_{2}, \ldots, j_{l} \in\{0, \ldots, 4\}$.

В дальнейшем $\alpha$-формулы над $T$ будем называть просто $\alpha$-формулами. Для $\alpha$-формулы $\Phi$ и переменной $x$ выражение $\Phi-x$ обозначает $\alpha$-формулу

$$
((\Phi+x)+x)+x \text {. }
$$

Пусть $\alpha$-формула $\Phi$ имеет вид (1) и переменная $z$ отлична от переменных $x_{i_{1}}, \ldots, x_{i_{l}}$. Положим

$$
\Phi^{-1}(z)=z \Phi^{-1}=\left(\left(\ldots\left(z h_{l}^{-1} / j_{l} x_{i_{l}}\right) h_{l-1}^{-1} / j_{l-1} x_{i_{l-1}} \ldots\right) h_{2}^{-1} / j_{2} x_{i_{2}}\right) h_{1}^{-1}-x_{i_{1}} .
$$

Если $\Psi-$ произвольная $\alpha$-формула, то запись $\Psi \Phi^{-1}$ обозначает $\alpha$-формулу

$$
\left(\left(\ldots\left(\Psi h_{l}^{-1} / j_{l} x_{i_{l}}\right) h_{l-1}^{-1} / j_{l-1} x_{i_{l-1}} \ldots\right) h_{2}^{-1} / j_{2} x_{i_{2}}\right) h_{1}^{-1}-x_{i_{1}},
$$

являющуюся результатом подстановки $\alpha$-формулы $\Psi$ вместо переменной $z$ в $\alpha$-формулу $\Phi^{-1}(z)$.

Лемма 2. Для любой $\alpha$-формулы $\Phi$ на множестве $E_{4}$ выполняется тождество

$$
\Phi \Phi^{-1}=0 .
$$


Доказательство. Пусть $\alpha$-формула Ф имеет вйд (1). Тогда

$$
\begin{aligned}
\Phi \Phi^{-1} & =\left(\left(\ldots\left(\left(\left(\ldots\left(x_{i_{1}} h_{1} \circ_{j_{2}} x_{i_{2}}\right) h_{2} \ldots \circ_{j_{l}} x_{i_{l}}\right) h_{l}\right) h_{l}^{-1} / j_{l} x_{i_{l}}\right) h_{l-1}^{-1} / j_{l-1} x_{i_{l-1}} \ldots\right) h_{2}^{-1} / j_{2} x_{i_{2}}\right) h_{1}^{-1}-x_{i_{1}} \\
& =\left(\left(\left(x_{i_{1}} h_{1} \circ_{j_{2}} x_{i_{2}}\right) h_{2}\right) h_{2}^{-1} / j_{2} x_{i_{2}}\right) h_{1}^{-1}-x_{i_{1}}=x_{i_{1}}-x_{i_{1}}=0 .
\end{aligned}
$$

Подстановки из $S_{4}$ будем записывать в виде циклов (транспозиций) или произведений (независимых) циклов. Подстановки умножаем слева направо в соответствии с правилом $\left(h_{i} h_{j}\right)(x)=h_{j}\left(h_{i}(x)\right)$. Для функции $f$ из $P_{4}(1)$ запись $f=[a b c d]$ означает, что $f(0)=a$, $f(1)=b, f(2)=c, f(3)=d$.

Лемма 3. Все одноместные функиии из $P_{4}$ реализуются $\alpha$-формулами над $T$.

Доказательство. Покажем, что для каждой функции $f$ из $P_{4}(1)$ существует $\alpha$-формула $\Phi$, которая реализует эту функцию. Для уменьшения числа проверок воспользуемся следующим замечанием. Две функции $f$ и $g$ из $P_{4}(1)$ назовем однотипными, если для некоторой подстановки $h$ из $S_{4}$ выполняется равенство

$$
f(x)=h(g(x))
$$

для любого $x \in E_{4}$. Ясно, что если одна из двух однотипных функций реализуется $\alpha-$ формулой над $T$, то и другая функщия реализуется $\alpha$-формулой над $T$. С учетом этого замечания достаточно построить $\alpha$-формулы для следующих 14 попарно неоднотипных функщий:

$$
\begin{aligned}
& f_{0}=[0000], \quad f_{1}=[0031], \quad f_{2}=[1310], \quad f_{3}=[0310], \quad f_{4}=[0332], \\
& f_{5}=[2131], \quad f_{6}=[0211], \quad f_{7}=[2223], \quad f_{8}=[1121], \quad f_{9}=[0100] \text {, } \\
& f_{10}=[3222], \quad f_{11}=[0011], \quad f_{12}=[0202], \quad f_{13}=[0330] \text {. }
\end{aligned}
$$

Определим подстановки из $S_{4}$ :

$$
\begin{aligned}
& h_{0}=(13), \quad h_{1}=(132), \quad h_{2}=(12), \quad h_{3}=(021), \quad h_{4}=(123) \text {, } \\
& h_{5}=(0132), \quad h_{6}=(23), \quad h_{7}=(031), \quad h_{8}=(013), \quad h_{9}=(03) \text {. }
\end{aligned}
$$

Легко проверить, что функция $f_{i}$ реализуется $\alpha$-формулой $\Phi_{i}, i=0,1, \ldots, 13$, где

$$
\begin{aligned}
& \Phi_{0}=x h_{0}+x, \quad \Phi_{1}=x h_{1}+x, \quad \Phi_{2}=\left(x h_{3}+x\right) h_{2}+x, \\
& \Phi_{3}=x h_{4}+x, \quad \Phi_{4}=x h_{2}+x, \quad \Phi_{5}=\left(x h_{5}+x\right) h_{2}+x, \\
& \Phi_{6}=x h_{6}+x, \quad \Phi_{7}=\left(x h_{7}+x\right) h_{6}+x, \quad \Phi_{8}=\left(x h_{8}+x\right) h_{6}+x, \\
& \Phi_{9}=\left(x h_{1}+x\right) \dot{h_{6}}+x, \quad \Phi_{10}=\left(x h_{9}+x\right) h_{2}+x, \quad \Phi_{11}=\left(x h_{2}+x\right) h_{6}+x, \\
& \Phi_{12}=x+x, \quad \Phi_{13}=\left(x h_{6}+x\right)+x \text {. }
\end{aligned}
$$

Лемма 3 доказана.

Через $\operatorname{Var}(\Phi)$ обозначим множество всех переменных, входящих в $\alpha$-формулу $\Phi$. Запись $\Phi\left(x_{1}, \ldots, x_{n}\right)$ означает, что $\operatorname{Var}(\Phi) \subseteq\left\{x_{1}, \ldots, x_{n}\right\}$. Значение $\alpha$-формулы $\Phi\left(x_{1}, \ldots, x_{n}\right)$ при значении переменных $x_{1}=a_{1}, \ldots, x_{n}=a_{n}$, где $a_{1}, \ldots, a_{n} \in E_{4}$, обозначим через $\Phi\left[\left(x_{1}, \ldots, x_{n}\right)=\left(a_{1}, \ldots, a_{n}\right)\right]$ или, короче, $\Phi\left(a_{1}, \ldots, a_{n}\right)$.

Теорема 1. Система функций $T \alpha$-полна, то есть

$$
[T]_{\alpha}=P_{4}
$$


Доказательство. Достаточно доказать включение

$$
f\left(x_{1}, \ldots, x_{n}\right) \in[T]_{\alpha}
$$

для любой функции $f \in P_{4}$. Утверждение (3) докажем индукцией по $n$.

При $n=1$ утверждение (3) верно в силу леммы 3. Допустим, что оно верно для всех $n<m$ и докажем его для $n=m$.

Следуя [1], покажем, что $[T]_{\alpha}$ содержит функции

$$
\psi_{a_{1}, \ldots, a_{m}, b}\left(x_{1}, \ldots, x_{m}\right)= \begin{cases}b, & \text { если }\left(x_{1}, \ldots, x_{m}\right)=\left(a_{1}, \ldots, a_{m}\right), \\ 0, & \text { если }\left(x_{1}, \ldots, x_{m}\right) \neq\left(a_{1}, \ldots, a_{m}\right)\end{cases}
$$

при любых $a_{1}, \ldots, a_{m}, b \in E_{4}, b \neq 0$.

Пусть $a_{1}, \ldots, a_{m} \in E_{4}$. Обозначим $a_{m}$ через $i$. Возьмем

$$
j= \begin{cases}0, & \text { если } i \text { нечетно, } \\ 1, & \text { если } i \text { четно. }\end{cases}
$$

Легко видеть, что $i \neq j$ и $2 i \neq 2 j$. Напомним, что элементы из $E_{4}$ мы умножаем (складываем, вычитаем) по модулю 4. Таким образом, $2 i \in E_{4}$ при любом $i \in E_{4}$.

Положим

$$
a=0, \quad b=i-j, \quad c=2, \quad d=j-i .
$$

Справедливы вхождения $a, b, c, d \in E_{4}$.

Из таблицы

\begin{tabular}{|c|c|cccc|c|}
\hline$i$ & $j$ & $a$ & $b$ & $c$ & $d$ & $j-b$ \\
\hline 0 & 1 & 0 & 3 & 2 & 1 & 2 \\
1 & 0 & 0 & 1 & 2 & 3 & 3 \\
2 & 1 & 0 & 1 & 2 & 3 & 0 \\
3 & 0 & 0 & 3 & 2 & 1 & 1 \\
\hline
\end{tabular}

видно, что элементы $a, b, c, d$ попарно различны при любом $i$, так что $E_{4}=\{a, b, c, d\}$.

Возьмем функщию

$$
f_{1}\left(x_{1}, \ldots, x_{m}\right)= \begin{cases}a ; & \text { если }\left(x_{1}, \ldots, x_{m-1}\right)=\left(a_{1}, \ldots, a_{m-1}\right), \\ b, & \text { если }\left(x_{1}, \ldots, x_{m-1}\right) \neq\left(a_{1}, \ldots, a_{m-1}\right),\end{cases}
$$

не зависящую существенно от $x_{m}$. По предположению индукции $f_{1} \in[T]_{\alpha}$. Пусть функция $f_{1}$ представляется $\alpha$-формулой

$$
\Phi_{1}=\left(\ldots\left(x_{i_{1}} h_{1} \circ_{j_{2}} x_{i_{2}}\right) h_{2} \cdots \circ_{j_{l}} x_{i_{l}}\right) h_{l},
$$

где $x_{i_{1}}, \ldots, x_{i_{l}} \in\left\{x_{1}, \ldots, x_{m-1}\right\}, h_{1}, \ldots, h_{l} \in S_{4}, j_{2}, \ldots, j_{l} \in\{0,1, \ldots, 4\}$.

В силу леммы 2 справедливо тождество

$$
\left(\left(\ldots\left(\Phi_{1} h_{l}^{-1} / j_{l} x_{i_{l}}\right) h_{l-1}^{-1} / j_{l-1} x_{i_{l-1}} \ldots\right) h_{2}^{-1} / j_{2} x_{i_{2}}\right) h_{1}^{-1}-x_{i_{1}}=0 .
$$

Определим подстановки $\tau_{1}=(i, j), \tau_{2}=(c, d)$ и рассмотрим функцию $\varphi\left(x_{1}, \ldots, x_{m}\right)$, представимую $\alpha$-формулой

$$
\Phi=\left(\left(\left(\Phi_{1}+x_{m}\right) \tau_{1}-x_{m}\right) \tau_{2}+x_{m}\right) \tau_{1}-x_{m} .
$$




\section{Справедливы равенства}

$$
\begin{aligned}
\varphi\left(a_{1}, \ldots, a_{m}\right) & =\varphi\left(a_{1}, \ldots, a_{m-1}, i\right) \\
& =\left(\left((a+i) \tau_{1}-i\right) \tau_{2}+i\right) \tau_{1}-i=\left(\left(i \tau_{1}-i\right) \tau_{2}+i\right) \tau_{1}-i \\
& =\left((j-i) \tau_{2}+i\right) \tau_{1}-i=(c+i) \tau_{1}-i=c+i-i=c .
\end{aligned}
$$

Далее,

$$
\begin{aligned}
\varphi\left(a_{1}, \ldots, a_{m-1}, j\right) & =\left(\left((a+j) \tau_{1}-j\right) \tau_{2}+j\right) \tau_{1}-j \\
& =\left((i-j) \tau_{2}+j\right) \tau_{1}-j=(i-j+j) \tau_{1}-j=j-j=0=a .
\end{aligned}
$$

Пусть $l \neq i, j$. Тогда

$$
\begin{aligned}
\varphi\left(a_{1}, \ldots, a_{m-1}, l\right) & =\left(\left((a+l) \tau_{1}-l\right) \tau_{2}+l\right) \tau_{1}-l \\
& =\left((l-l) \tau_{2}+l\right) \tau_{1}-l=l \tau_{1}-l=l-l=0=a .
\end{aligned}
$$

Пусть $\left(b_{1}, \ldots, b_{m-1}\right) \neq\left(a_{1}, \ldots, a_{m-1}\right), l \in E_{4}$. Рассмотрим три случая.

1. Пусть $b+l=i$. Тогда $l=i-b=j$.

Справедливы равенства

$$
\begin{aligned}
\varphi\left(b_{1}, \ldots, b_{m-1}, l\right) & =\varphi\left(b_{1}, \ldots, b_{m-1}, j\right) \\
& =\left(\left((b+j) \tau_{1}-j\right) \tau_{2}+j\right) \tau_{1}-j \\
& =\left((j-j) \tau_{2}+j\right) \tau_{1}-j=j \tau_{1}-j=i-j=b .
\end{aligned}
$$

2. Пусть $b+l=j$. В этом случае $l=j-b$ и $d+l=2 i+3 j \notin\{i, j\}$.

Справедливы равенства

$$
\begin{aligned}
\varphi\left(b_{1}, \ldots, b_{m-1}, l\right) & =\left(\left((b+l) \tau_{1}-l\right) \tau_{2}+l\right) \tau_{1}-l=\left((i-l) \tau_{2}+l\right) \tau_{1}-l \\
& =\left(c \tau_{2}+l\right) \tau_{1}-l=(d+l) \tau_{1}-l=d+l-l=d .
\end{aligned}
$$

3. Пусть $b+l \neq i, j$. Тогда

$$
\begin{aligned}
\varphi\left(b_{1}, \ldots, b_{m-1}, l\right) & =\left(\left((b+l) \tau_{1}-l\right) \tau_{2}+l\right) \tau_{1}-l \\
& =\left((b+l-l) \tau_{2}+l\right) \tau_{1}-l=(b+l) \tau_{1}-l=b+l-l=b .
\end{aligned}
$$

Таким образом, $\varphi \in[T]_{\alpha}$ и

$$
\varphi\left(x_{1}, \ldots, x_{m}\right)= \begin{cases}c, & \text { если }\left(x_{1}, \ldots, x_{m}\right)=\left(a_{1}, \ldots, a_{m}\right), \\ a, & \text { если }\left(x_{1}, \ldots, x_{m-1}\right)=\left(a_{1}, \ldots, a_{m-1}\right) \text { и } x_{m} \neq a_{m}, \\ b, & \text { если }\left(x_{1}, \ldots, x_{m-1}\right) \neq\left(a_{1}, \ldots, a_{m-1}\right) \text { и } x_{m} \neq j-b, \\ d, & \text { если }\left(x_{1}, \ldots, x_{m-1}\right) \neq\left(a_{1}, \ldots, a_{m-1}\right) \text { и } x_{m}=j-b .\end{cases}
$$

Рассмотрим функцию

$$
\psi\left(x_{1}, \ldots, x_{m}\right)=\varphi\left(x_{1}, \ldots, x_{m}\right) \circ_{i} x_{m} .
$$

Ясно, что $\psi \in[T]_{\alpha}$. Покажем, что для $\psi$ выполняется соотношение

$$
\psi\left(x_{1}, \ldots, x_{m}\right)= \begin{cases}c, & \text { если }\left(x_{1}, \ldots, x_{m}\right)=\left(a_{1}, \ldots, a_{m}\right), \\ f_{1}\left(x_{1}, \ldots, x_{m}\right) & \text { в остальных случаях }\end{cases}
$$


Действительно, пусть $i=0\left(i=a_{m}\right)$. Тогда $j-b=2, a=0, b=3, c=2, d=1$. Справедливы равенства

$$
\begin{array}{rlrl}
c \circ_{0} i & =c, \quad a \circ_{0} l=a, & l \neq i, \\
d \circ_{0}(j-b) & =1 \circ_{0} 2=3=b, & & \\
b \circ_{0} l & =3 \circ_{0} l=3=b, & & l \neq j-b .
\end{array}
$$

Следовательно,

$$
\psi\left(x_{1}, \ldots, x_{m}\right)= \begin{cases}c, & \text { если }\left(x_{1}, \ldots, x_{m}\right)=\left(a_{1}, \ldots, a_{m}\right), \\ a, & \text { если }\left(x_{1}, \ldots, x_{m-1}\right)=\left(a_{1}, \ldots, a_{m-1}\right) \text { и } x_{m} \neq a_{m}, \\ b, & \text { если }\left(x_{1}, \ldots, x_{m-1}\right) \neq\left(a_{1}, \ldots, a_{m-1}\right) .\end{cases}
$$

Таким образом, соотношение (6) верно, когда $i=0$.

Случаи $i=1,2,3$ проверяются аналогично.

Пусть $\Psi\left(x_{1}, \ldots, x_{m}\right)-\alpha$-формула, реализующая функцию $\psi$. Рассмотрим $\alpha$-формулу

$$
\Delta=\Psi \Phi_{1}^{-1}=\left(\left(\ldots\left(\Psi h_{l}^{-1} / j_{l} x_{i_{l}}\right) h_{l-1}^{-1} / j_{l-1} x_{i_{l-1}} \ldots\right) h_{2}^{-1} / j_{2} x_{i_{2}}\right) h_{1}^{-1}-x_{i_{1}},
$$

где $h_{1}^{-1}, \ldots, h_{l}^{-1}, x_{i_{1}}, \ldots, x_{i_{l}}, j_{2}, \ldots, j_{l}$ взяты из тождества (5).

Ясно, что

$$
\Delta\left(a_{1}, \ldots, a_{m}\right)=\left(\left(\ldots\left(c h_{l}^{-1} / j_{l} a_{i_{l}}\right) \ldots\right) h_{2}^{-1} / j_{2} a_{i_{2}}\right) h_{1}^{-1}-a_{i_{1}}=c_{1}
$$

для некоторого $c_{1} \in E_{4}$. Пусть $l \neq a_{m}$. Ввиду (5)

$$
\begin{aligned}
\Delta\left(a_{1}, \ldots, a_{m-1}, l\right) & =\left(\left(\ldots\left(a h_{l}^{-1} / j_{l} a_{i_{l}}\right) \ldots\right) h_{2}^{-1} / j_{2} a_{i_{2}}\right) h_{1}^{-1}-a_{i_{1}} \\
& =\left(\left(\ldots\left(0 h_{l}^{-1} / j_{l} a_{i_{l}}\right) \ldots\right) h_{2}^{-1} / j_{2} a_{i_{2}}\right) h_{1}^{-1}-a_{i_{1}}=0 .
\end{aligned}
$$

Отображение

$$
x \mapsto\left(\left(\ldots\left(x h_{l}^{-1} / j_{l} a_{i_{l}}\right) \ldots\right) h_{2}^{-1} / j_{2} a_{i_{2}}\right) h_{1}^{-1}-a_{i_{1}}
$$

биективно на $E_{4}$. Поэтому $c_{1} \neq 0$.

Пусть $\left(b_{1}, \ldots, b_{m-1}\right) \neq\left(a_{1}, \ldots, a_{m-1}\right)$. Учитывая (5), получаем, что

$$
\Delta\left(b_{1}, \ldots, b_{m}\right)=\left(\left(\ldots\left(b h_{l}^{-1} / j_{l} b_{i_{l}}\right) \ldots\right) h_{2}^{-1} / j_{2} b_{i_{2}}\right) h_{1}^{-1}-b_{i_{1}}=0 .
$$

Таким образом, $\alpha$-формула $\Delta$ реализует функцию $\psi_{a_{1}, \ldots, a_{m}, c_{1}}$, то есть

$$
\psi_{a_{1}, \ldots, a_{m}, c_{1}} \in[T]_{\alpha} .
$$

Поскольку $[T]_{\alpha}$ содержит все функции вида $h\left(\psi_{a_{1}, \ldots, a_{m}, c_{1}}\right)$, где $h \in S_{4}$, множество $[T]_{\alpha}$ содержит и все функции вида (4).

С помощью функщий вида $\psi_{a_{1}, \ldots, a_{m}, b}$ можно (см. [1]) из любой одной функции $f\left(x_{1}, \ldots, x_{m}\right) \in[T]_{\alpha}$ построить любую функцию $\varphi\left(x_{1}, \ldots, x_{m}\right) \in P_{4}$, не выходя из класса $[T]_{\alpha}$. Действительно, если функщия $f$ представляется $\alpha$-формулой

$$
\left(\left(\ldots\left(x_{j_{1}} g_{1} \circ_{i_{2}} x_{j_{2}}\right) g_{2} \circ_{i_{3}} \ldots\right) g_{t-1} \circ_{i_{t}} x_{j_{t}}\right) g_{t}
$$

то функщия

$$
\left(\left(\ldots\left(\left(\psi_{a_{1}, \ldots, a_{m}, b}+x_{j_{1}}\right) g_{1} \circ_{i_{2}} x_{j_{2}}\right) g_{2} \circ_{i_{3}} \ldots\right) g_{t-1} \circ_{i_{t}} x_{j_{t}}\right) g_{t}
$$


содержится в $[T]_{\alpha}$ и отличается от $f$ лишь в точке $\left(a_{1}, \ldots, a_{m}\right)$, в которой при подходящем $b(b \neq 0)$ принимает любое значение, отличное от $f\left(a_{1}, \ldots, a_{m}\right)$. Это вытекает из того, что отображение

$$
\sigma: x \mapsto\left(\left(\ldots\left(\left(x+a_{j_{1}}\right) g_{1} \circ_{i_{2}} a_{j_{2}}\right) g_{2} \circ_{i_{3}} \ldots\right) g_{t-1} \circ_{i_{t}} a_{j_{t}}\right) g_{t}
$$

биективно на множестве $E_{4}$ и $\sigma(0)=f\left(a_{1}, \ldots, a_{m}\right)$.

Изменив таким образом значение функци $f$ в нужном числе точек на подходящие элементы из $E_{4}$, мы сможем получить любую функцию от $m$ переменных из $P_{4}$. Следовательно, включение (3) верно для $n=m$.

Теорема 1 доказана.

Обозначим через $\mu(f)$ арность операции $f \in P_{k}$. Говорят, что алгебры

$$
A=\left(E_{k},\left\{f_{i} \mid i \in I\right\}\right), \quad B=\left(E_{k},\left\{g_{i} \mid i \in I\right\}\right)
$$

имеют один и тот же тип $\tau=\left\{n_{i} \mid i \in I\right\}$ (являются однотипными), если

$$
\mu\left(f_{i}\right)=\mu\left(g_{i}\right)=n_{i}
$$

для всех $i \in I$. При этом операции $f_{i}$ и $g_{i}$, отвечающие одному и тому же индексу $i \in I$, называются одноименными.

Лемма 4. Пусть $\varphi-$ изоморфизм алгебры $A=\left(E_{k},\left\{f_{i} \mid i \in I\right\}\right)$ на однотипную алгебру $B=\left(E_{k},\left\{g_{i} \mid i \in I\right\}\right), F=\left\{f_{i} \mid i \in I\right\}, G=\left\{g_{i} \mid i \in I\right\}$. Тогдa

$$
f\left(x_{1}, \ldots, x_{n}\right) \in[F]_{\alpha} \Longleftrightarrow \varphi\left(f\left(\varphi^{-1}\left(x_{1}\right), \ldots, \varphi^{-1}\left(x_{n}\right)\right)\right) \in[G]_{\alpha}
$$

для любой функции $f\left(x_{1}, \ldots, x_{n}\right)$ из $P_{k}$.

Доказательство. Для записи $\alpha$-формул над $F$ будем использовать сигнатуру $\left\{f_{i} \mid i \in I\right\}$, а для записи $\alpha$-формул над $G$ - сигнатуру $\left\{g_{i} \mid i \in I\right\}$. Если нужно подчеркнуть различие между функщией $f_{i}$ из $F$ и соответствующим ей функциональным символом $f_{i}$, то функцию $f_{i}$ будем обозначать через $f_{i}^{A}$.

Пусть $\Phi$ - произвольная $\alpha$-формула над $F$. Через $\Phi^{*}$ обозначим $\alpha$-формулу над $G$, которая получается из $\Phi$ заменой символов $f_{i}$ на соответствующие им символы $g_{i}, i \in I$. Формулу $\Phi^{*}$ назовем переводом формулы $\Phi$.

Отображение $\varphi$ является подстановкой на множестве $E_{k}$ и

$$
\varphi\left(f_{i}^{A}\left(a_{1}, \ldots, a_{n_{i}}\right)\right)=g_{i}^{B}\left(\varphi\left(a_{1}\right), \ldots, \varphi\left(a_{n_{i}}\right)\right)
$$

для всех $a_{1}, \ldots, a_{n_{i}}$ из $E_{k}$ и для всех $i$ из $I\left(n_{i}=\mu\left(f_{i}\right)\right)$. Индукщией по построению $\alpha$-формулы над $F$ устанавливается равенство

$$
\varphi\left(\Phi\left(a_{1}, \ldots, a_{n}\right)\right)=\Phi^{*}\left(\varphi\left(a_{1}\right), \ldots, \varphi\left(a_{n}\right)\right)
$$

для всех $a_{1}, \ldots, a_{n}$ из $E_{k}$. Отсюда

$$
\begin{aligned}
\Phi\left(a_{1}, \ldots, a_{n}\right) & =\varphi^{-1}\left(\Phi^{*}\left(\varphi\left(a_{1}\right), \ldots, \varphi\left(a_{n}\right)\right)\right) \\
\Phi^{*}\left(a_{1}, \ldots, a_{n}\right) & =\varphi\left(\Phi\left(\varphi^{-1}\left(a_{1}\right), \ldots, \varphi^{-1}\left(a_{n}\right)\right)\right) .
\end{aligned}
$$


Пусть $f\left(x_{1}, \ldots, x_{n}\right) \in[F]_{\alpha}$ и $\Phi\left(x_{1}, \ldots, x_{n}\right)-\alpha$-формула над $F$, реализующая $f$. Перевод $\Phi^{*}\left(x_{1}, \ldots, x_{n}\right)$ формулы $\Phi$ является $\alpha$-формулой над $G$ и реализует некоторую функиию $g\left(x_{1}, \ldots, x_{n}\right)$. Для всех $a_{1}, \ldots, a_{n}$ из $E_{k}$

$$
\begin{aligned}
g\left(a_{1}, \ldots, a_{n}\right) & =\Phi^{*}\left(a_{1}, \ldots, a_{n}\right)=\varphi\left(\Phi\left(\varphi^{-1}\left(a_{1}\right), \ldots, \varphi^{-1}\left(a_{n}\right)\right)\right) \\
& =\varphi\left(f\left(\varphi^{-1}\left(a_{1}\right), \ldots, \varphi^{-1}\left(a_{n}\right)\right)\right) .
\end{aligned}
$$

Это означает, что

$$
g\left(x_{1}, \ldots, x_{n}\right)=\varphi\left(f\left(\varphi^{-1}\left(x_{1}\right), \ldots, \varphi^{-1}\left(x_{n}\right)\right)\right)
$$

и $\varphi\left(f\left(\varphi^{-1}\left(x_{1}\right), \ldots, \varphi^{-1}\left(x_{n}\right)\right)\right) \in[G]_{\alpha}$.

Пусть теперь $\varphi\left(f\left(\varphi^{-1}\left(x_{1}\right), \ldots, \varphi^{-1}\left(x_{n}\right)\right)\right) \in[G]_{\alpha}$ и $\Psi\left(x_{1}, \ldots, x_{n}\right)-\alpha$-формула над $G$, реализующая функщию

$$
g\left(x_{1}, \ldots, x_{n}\right)=\varphi\left(f\left(\varphi^{-1}\left(x_{1}\right), \ldots, \varphi^{-1}\left(x_{n}\right)\right)\right) .
$$

Формула $\Psi$ является переводом некоторой $\alpha$-формулы $\Phi\left(x_{1}, \ldots, x_{n}\right)$ над $F$, то есть $\Psi=\Phi^{*}$. Для функции $f_{1}\left(x_{1}, \ldots, x_{n}\right)$, реализуемой $\alpha$-формулой $\Phi$ над $F$,

$$
\begin{aligned}
f_{1}\left(a_{1}, \ldots, a_{n}\right) & =\Phi\left(a_{1}, \ldots, a_{n}\right)=\varphi^{-1}\left(\Phi^{*}\left(\varphi\left(a_{1}\right), \ldots, \varphi\left(a_{n}\right)\right)\right) \\
& =\varphi^{-1}\left(\Psi\left(\varphi\left(a_{1}\right), \ldots, \varphi\left(a_{n}\right)\right)\right)=\varphi^{-1}\left(g\left(\varphi\left(a_{1}\right), \ldots, \varphi\left(a_{n}\right)\right)\right) \\
& =\varphi^{-1}\left(\varphi\left(f\left(\varphi^{-1} \varphi\left(a_{1}\right), \ldots, \varphi^{-1} \varphi\left(a_{n}\right)\right)\right)\right)=f\left(a_{1}, \ldots, a_{n}\right)
\end{aligned}
$$

для всех $a_{1}, \ldots, a_{n}$ из $E_{k}$. Следовательно, $f\left(x_{1}, \ldots, x_{n}\right)$ реализуется $\alpha$-формулой $\Phi\left(x_{1}, \ldots, x_{n}\right)$ и $f\left(x_{1}, \ldots, x_{n}\right) \in[F]_{\alpha}$. Лемма 4 доказана.

Из леммы 4 непосредственно вытекает следующее утверждение.

Теорема 2. Если однотипные алгебры $A=\left(E_{k},\left\{f_{i} \mid i \in I\right\}\right)$ u $B=\left(E_{k},\left\{g_{i} \mid i \in I\right\}\right)$ изоморфны, то система функций $F=\left\{f_{i} \mid i \in I\right\} \alpha$-полна тогда и только тогда, когда $\alpha$-полна система функиий $G=\left\{g_{i} \mid i \in I\right\}$, то есть

$$
[F]_{\alpha}=P_{k} \Longleftrightarrow[G]_{\alpha}=P_{k}
$$

Согласно [1] для любой квазигруппы $\left(E_{4}, \cdot\right)$ может быть построена изотопная ей лупа $\left(E_{4}\right.$, о) с нейтральным элементом 0 и такая, что

$$
\left[x_{1} x_{2}, S_{4}\right]_{\alpha}=\left[x_{1} \circ x_{2}, S_{4}\right]_{\alpha} \text {. }
$$

При этом операция о определяется $\alpha$-формулой вида

$$
x \circ y=\left(x h_{1} \cdot y\right) h_{2} \text {, }
$$

где $h_{1}, h_{2} \in S_{4}$. Если операция - имеет таблицу Кэли

\begin{tabular}{c|cccc}
$\cdot$ & 0 & 1 & 2 & 3 \\
\hline 0 & $a_{00}$ & $a_{01}$ & $a_{02}$ & $a_{03}$ \\
1 & $a_{10}$ & $a_{11}$ & $a_{12}$ & $a_{13}$ \\
2 & $a_{20}$ & $a_{21}$ & $a_{22}$ & $a_{23}$ \\
3 & $a_{30}$ & $a_{31}$ & $a_{32}$ & $a_{33}$,
\end{tabular}


To

$$
h_{1}=\left(\begin{array}{cccc}
0 & 1 & 2 & 3 \\
i_{0} & i_{1} & i_{2} & i_{3}
\end{array}\right)
$$

где $i_{0}$ определяется из условия $a_{i_{0}, 0}=0, \mathrm{a} i_{1}, i_{2}, i_{3}-$ из условий $a_{i_{1}, 0}=a_{i_{0}, 1}, a_{i_{2}, 0}=a_{i_{0}, 2}$, $a_{i_{3}, 0}=a_{i_{0}, 3}$ соответственно.

Подстановка $h_{2}$ задается равенством

$$
h_{2}=\left(\begin{array}{cccc}
0 & a_{i_{0}, 1} & a_{i_{0}, 2} & a_{i_{0}, 3} \\
0 & 1 & 2 & 3
\end{array}\right)
$$

Таблища Кэли для операции о имеет вид

\begin{tabular}{c|cccc}
$\circ$ & 0 & 1 & 2 & 3 \\
\hline 0 & 0 & 1 & 2 & 3 \\
1 & 1 & $b_{11}$ & $b_{12}$ & $b_{13}$ \\
2 & 2 & $b_{21}$ & $b_{22}$ & $b_{23}$ \\
3 & 3 & $b_{31}$ & $b_{32}$ & $b_{33}$
\end{tabular}

С помощью подстановки $h_{1}$ происходит перестановка строк во внутренней части таблищы (7), а с помощью подстановки $h_{2}$ - переименование элементов.

На множестве $E_{4}$ можно задать всего четыре лупы с таблицей Кэли вида (10):

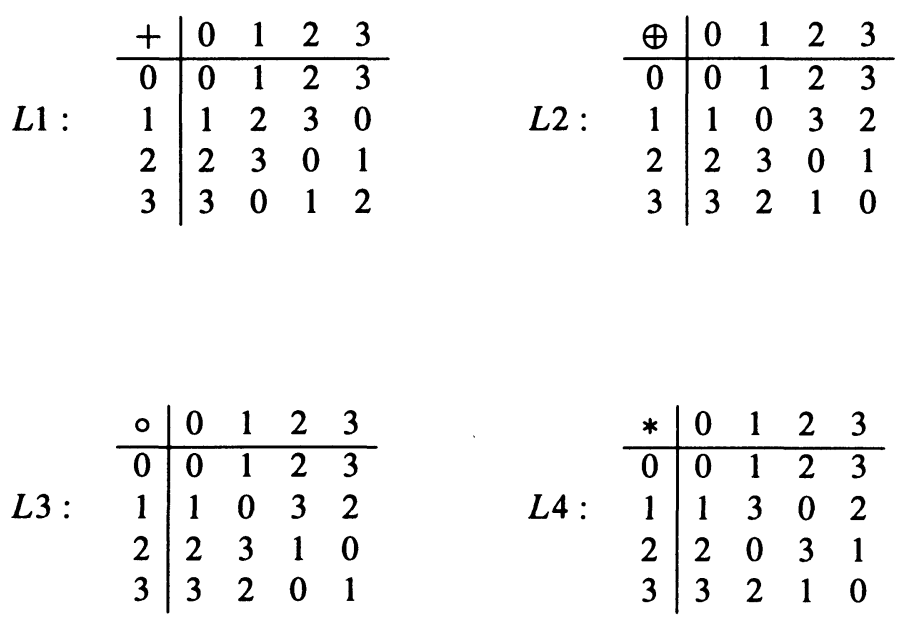

Все эти лупы являются коммутативными и ассоциативными лупами, то есть абелевыми группами. Группы $\left(E_{4},+\right),\left(E_{4}, \circ\right),\left(E_{4}, *\right)$ - циклические группы, изоморфные группе $\mathbf{Z}_{4}$, а группа $\left(E_{4}, \oplus\right)$ - элементарная 2-группа, изоморфная прямой сумме $\mathbf{Z}_{2}+\mathbf{Z}_{2}$.

Согласно [1] для группы $\left(E_{4}, \oplus\right)$ система функций $T_{2}=S_{4} \cup\left\{x_{1} \oplus x_{2}\right\}$ не является $\alpha$-полной. Вопрос об $\alpha$-полноте системы

$$
T_{2}^{\prime}=S_{4} \cup\left\{\oplus, \circ_{0}, \circ_{1}, \circ_{2}, \circ_{3}\right\}
$$

остается открытым. Известно лишь, что все одноместные функщия представимы $\alpha$ формулами над $T_{2}^{\prime}$.

Занумеруем подстановки из $S_{4}$ произвольным образом. Получим

$$
S_{4}=\left\{h_{i} \mid i \in I\right\},
$$


где $I=\{1,2, \ldots, 24\}$. Подстановка $\varphi=(12)$ является изоморфизмом групты $\left(E_{4},+\right)$ на группу $\left(E_{4}\right.$, o), а подстановка $\psi=(23)$ - изоморфизмом группы $\left(E_{4},+\right)$ на группу $\left(E_{4}, *\right)$. Положим

$$
\begin{aligned}
h_{i}^{B} & =\varphi^{-1} h_{i} \varphi, & & i \in I, \\
x \circ_{j}^{B} y & =\varphi\left(\varphi^{-1} x \circ_{j} \varphi^{-1} y\right), & & j \in\{0,1,2,3\}, \\
h_{i}^{C} & =\psi^{-1} h_{i} \psi, & & i \in I, \\
x \circ_{j}^{C} y & =\psi\left(\psi^{-1} x \circ_{j} \psi^{-1} y\right), & & j \in\{0,1,2,3\} .
\end{aligned}
$$

Тогда $\varphi$ - изоморфизм алгебры

$$
A=\left(E_{4},+,\left\{h_{i} \mid i \in I\right\},\left\{o_{j} \mid j \in\{0,1,2,3\}\right\}\right)
$$

на алгебру

$$
B=\left(E_{4}, \circ,\left\{h_{i}^{B} \mid i \in I\right\},\left\{0_{j}^{B} \mid j \in\{0,1,2,3\}\right\}\right),
$$

а $\psi$ - изоморфизм алгебры $A$ на алгебру

$$
C=\left(E_{4}, *,\left\{h_{i}^{C} \mid i \in I\right\},\left\{0_{j}^{C} \mid j \in\{0,1,2,3\}\right\}\right) .
$$

Лемма 5. Имеют место равенства

$$
\begin{aligned}
& {\left[S_{4} \cup\left\{\circ_{0}, \circ_{1}, \circ_{2}, \circ_{3}\right\}\right]_{\alpha}=\left[S_{4} \cup\left\{\circ_{0}^{B}, \circ_{1}^{B}, \circ_{2}^{B}, \circ_{3}^{B}\right\}\right]_{\alpha},} \\
& {\left[S_{4} \cup\left\{\circ_{0}, \circ_{1}, \circ_{2}, \circ_{3}\right\}\right]_{\alpha}=\left[S_{4} \cup\left\{\circ_{0}^{C}, \circ_{1}^{C}, \circ_{2}^{C}, \circ_{3}^{C}\right\}\right]_{\alpha} .}
\end{aligned}
$$

Доказательство. Легко видеть, что верны тождества

$$
\begin{array}{ll}
x \circ_{0} \varphi(y)=x \circ_{3} y, & x \circ_{1} \varphi(y)=x \circ_{1} y, \\
x \circ_{2} \varphi(y)=x \circ_{2} y, & x \circ_{3} \varphi(y)=x \circ_{0} y .
\end{array}
$$

Отсюда,

$$
\begin{aligned}
& x \circ_{0}^{B} y=\left(x \varphi \circ_{0} y \varphi\right) \varphi=\left(x \varphi \circ_{3} y\right) \varphi, \\
& x \circ_{3}^{B} y=\left(x \varphi \circ_{3} y \varphi\right) \varphi=\left(x \varphi \circ_{0} y\right) \varphi, \\
& x \circ_{j}^{B} y=\left(x \varphi \circ_{j} y \varphi\right) \varphi=\left(x \varphi \circ_{j} y\right) \varphi,
\end{aligned}
$$

если $j=1,2$. Во всех случаях операция ${ }_{j}^{B}, j \in\{0,1,2,3\}$, представима $\alpha$-формулой над $S_{4} \cup\left\{o_{0}, \circ_{1}, o_{2}, o_{3}\right\}$. Очевидно, что верно и обратное: каждая операция о ется $\alpha$-формулой над системой $S_{4} \cup\left\{\circ_{0}^{B}, \circ_{1}^{B}, \circ_{2}^{B}, \circ_{3}^{B}\right\}$. Первое равенство доказано. Второе равенство доказывается аналогично с заменой $\varphi$ на $\psi$.

Из леммы 5 и теоремы 2 получаем $\alpha$-полноту систем функций

$$
T_{3}=S_{4} \cup\left\{\circ, \circ_{0}, \circ_{1}, \circ_{2}, \circ_{3}\right\}, \quad T_{4}=S_{4} \cup\left\{*, \circ_{0}, \circ_{1}, \circ_{2}, \circ_{3}\right\},
$$

где $\circ$ и $*$ - бинарные операции с таблицами $L 3$ и $L 4$ соответственно. 
Теорема 3. Если $\left(E_{4}, \cdot\right)-$ произвольная квазигруппа, изотопная циклической группе $\mathbf{Z}_{4}$, то система функций $S_{4} \cup\left\{\cdot, \circ_{0}, \circ_{1}, \circ_{2}, \circ_{3}\right\}$ является $\alpha$-полной.

Доказательство. Пусть квазигруппа $\left(E_{4}, \cdot\right)$ имеет таблицу Кэли (7). Используя подстановки $(8)$ и (9), построим изотопную лупу $\left(E_{4}, \diamond\right)$ с нейтральным элементом 0 и операцией

$$
x \diamond y=\left(x h_{1} \cdot y\right) h_{2} \text {. }
$$

Лупа $\left(E_{4}, \diamond\right)$ изотопна группе $\mathbf{Z}_{4}$. По теореме Алберта (см. [3], с. 17) лупа $\left(E_{4}, \diamond\right)$ является группой, изоморфной группе $\mathbf{Z}_{4}$. Поэтому операция $\diamond$ совпадает с одной из операций, которые задаются таблицами $L 1, L 3, L 4$. Отсюда следует $\alpha$-полнота системы функций $S_{4} \cup\left\{\diamond, \circ_{0}, \circ_{1}, \circ_{2}, \circ_{3}\right\}$. В силу (11)

$$
\left[S_{4} \cup\left\{\cdot, \circ_{0}, \circ_{1}, \circ_{2}, \circ_{3}\right\}\right]_{\alpha}=\left[S_{4} \cup\left\{\diamond, \circ_{0}, \circ_{1}, \circ_{2}, \circ_{3}\right\}\right]_{\alpha}=P_{4} \text {. }
$$

Теорема 3 доказана.

Перейдем теперь к случаю $k=3$. Определим на множестве $E_{3}$ бинарные операции $\circ_{i}, i=0,1,2$, полагая

$$
\begin{aligned}
& 1 \circ_{0} 1=2, \quad 2 \circ_{0} 1=1, \quad 0 \circ_{0} 2=2, \quad 2 \circ_{0} 2=0, \\
& 0 \circ_{1} 0=2, \quad 2 \circ_{1} 0=0, \quad 1 \circ_{1} 2=2, \quad 2 \circ_{1} 2=1 \text {, } \\
& 1 \circ_{2} 0=2, \quad 2 \circ_{2} 0=1, \quad 0 \circ_{2} 1=2, \quad 2 \circ_{2} 1=0
\end{aligned}
$$

и $a \circ_{i} b=a$ в остальных случаях.

Пусть

$$
T=S_{3} \cup\left\{+, \circ_{0}, \circ_{1}, \circ_{2}\right\} .
$$

Легко проверить, что все одноместные функции из $P_{3}$ реализуются $\alpha$-формулами над $T$.

Теорема 4. Система функиий $T \alpha$-полна, то есть $[T]_{\alpha}=P_{3}$.

Доказательство аналогично доказательству теоремы 1.

На множестве $E_{3}$ можно задать всего одну лупу $\left(E_{3}\right.$, о) с нейтральным элементом 0 . В этой лупе операция о совпадает с операцией сложения по модулю 3. Любая квазигруппа $\left(E_{3}, \cdot\right)$ изотопна лупе $\left(E_{3}, \circ\right)$, причем согласно [1] имеет место равенство

$$
x \circ y=\left(x h_{1} \cdot y\right) h_{2},
$$

где $h_{1}, h_{2}$ - подходящие подстановки из $S_{3}$. Отсюда и из теоремы 4 получаем следующее утверждение.

Теорема 5. Если $\left(E_{3}, \cdot\right)-$ произвольная квазигруппа, то система функций $S_{3} \cup\left\{\cdot, \circ_{0}, \circ_{1}, \circ_{2}\right\}$ является $\alpha$-полной.

\section{Список литературы}

1. Глухов М. М., Об $\alpha$-замкнутых классах и $\alpha$-полных системах функций $k$-значной логики. Дискретиая математика (1989) 1, №1, 16-21.

2. Чернышов А. Л., Условия $\alpha$-полноты систем функций многозначной логики. Дискретная математика (1992) 4, №4, 117-130.

3. Белоусов В. Д., Осповы теории квазигрупп и луп. Наука, Москва, 1967.

4. Яблонский С. В., Введение в дискретиую математику. Наука, Москва, 1979.

Статья поступила 17.08.2004.

Переработанный вариант поступил 27.06.2005. 